\title{
Consensus provisional definition of agitation in cognitive disorders
}

Jeffrey Cummings, Jacobo Mintzer, Henry Brodaty, Mary Sano, Sube Banerjee, D. P. Devanand, Serge Gauthier, Robert Howard, Krista Lanctôt, Constantine G. Lyketsos, Elaine Peskind, Anton P. Porsteinsson, Edgardo Reich, Cristina Sampaio, David Steffens, Marc Wortmann, Kate Zhong

\section{Source}

Jeffrey Cummings, Jacobo Mintzer, Henry Brodaty, Mary Sano, Sube Banerjee, D.P. Devanand. (2014). Agitation in cognitive disorders: International Psychogeriatric Association provisional consensus clinical and research definition. Int. Psychogeriatr., vol. $27(1), 7-17$.

A. The patient meets criteria for a cognitive impairment or dementia syndrome (e.g. AD, FTD, DLB, vascular dementia, other dementias, a pre-dementia cognitive impairment syndrome such as mild cognitive impairment or other cognitive disorder).

B. The patient exhibits at least one of the following behaviors that are associated with observed or inferred evidence of emotional distress (e.g. rapid changes in mood, irritability, outbursts). The behavior has been persistent or frequently recurrent for a minimum of two weeks' and represents a change from the patient's usual behavior.

(a) Excessive motor activity (examples include: pacing, rocking, gesturing, pointing fingers, restlessness, performing repetitious mannerisms).

(b) Verbal aggression (e.g. yelling, speaking in an excessively loud voice, using profanity, screaming, shouting).

(c) Physical aggression (e.g. grabbing, shoving, pushing, resisting, hitting others, kicking objects or people, scratching, biting, throwing objects, hitting self, slamming doors, tearing things, and destroying property).

C. Behaviors are severe enough to produce excess disability, which in the clinician's opinion is beyond that due to the cognitive impairment and including at least one of the following:

(a) Significant impairment in interpersonal relationships.

(b) Significant impairment in other aspects of social functioning. 
(c) Significant impairment in ability to perform or participate in daily living activities.

D. While co-morbid conditions may be present, the agitation is not attributable solely to another psychiatric disorder, suboptimal care conditions, medical condition, or the physiological effects of a substance. 